\title{
Seasonal Variation in Diverticulitis: Evidence From Both Hemispheres
}

Joel T. Adler, M.D., M.P.H. ${ }^{1,2}$, David C. Chang, Ph.D., M.B.A., M.P.H. ${ }^{2}$, Andrew T. Chan, M.D., M.P.H. ${ }^{3}$, Omar Faiz, M.B.B.S., M.S. ${ }^{4,5}$, and Lillias H. Maguire, M.D. ${ }^{2}$

${ }^{1}$ Center for Surgery and Public Health at Brigham and Women's Hospital, Boston, Massachusetts

${ }^{2}$ Department of Surgery, Massachusetts General Hospital, Boston, Massachusetts

${ }^{3}$ Division of Gastroenterology, Department of Medicine, Massachusetts General Hospital, Boston, Massachusetts

${ }^{4}$ St Mark's Hospital, North West London NHS Trust, London, United Kingdom

${ }^{5}$ Imperial College London, London, United Kingdom

\section{Abstract}

BACKGROUND: Diverticulitis is a common and morbid disease with incompletely understood risk factors and pathophysiology. Geographic and, recently, seasonal trends in diverticulitis have been described in the United States.

OBJECTIVE: The purpose of this study was to investigate and compare seasonal trends in urgent hospital admission for diverticulitis in geographically distinct populations in the northern and southern hemispheres.

DESIGN: Inpatient, urgent admissions for diverticulitis were identified within the Dr Foster Intelligence Global Comparators Dataset, a global benchmarking collaborative.

SETTINGS: Admissions to participating hospitals in the United Kingdom, Australia, and the United States were identified between 2008 and 2013.

PATIENTS: A total of 18,672 urgent admissions for diverticulitis were identified among 5.5million admissions.

MAIN OUTCOME MEASURES: Four separate hypothesis testing methods were used to identify seasonal trends in diverticulitis admissions among international patient populations.

RESULTS: Seasonal trends were present in all 3 countries. A summer peak was observed in both hemispheres using multiple statistical testing methods. Logistic regression analyses identified summer months as significantly associated with diverticulitis admission in all 3 countries.

LIMITATIONS: This study is limited by restriction to inpatient admissions, reliance on administrative data, and participation of select hospitals within the database.

Correspondence: Lillias H. Maguire, M.D., Department of Surgery, Massachusetts General Hospital, 55 Fruit St, Boston, MA 02114. lhmaguire@partners.org.

Financial Disclosure: None reported. 
CONCLUSIONS: These data suggest a shared seasonal risk factor among geographically distinct populations for diverticulitis.

\section{Keywords}

Diverticulitis; Seasonal variation

Diverticulitis is a common disease with an incompletely understood natural history and pathophysiology. More than 200,000 people are hospitalized yearly in the United States for diverticulitis and its attendant risks of perforation, abscess, fistula, and surgery. ${ }^{1}$ Geographic variation in diverticulitis has long been appreciated. ${ }^{2}$ The precursor lesion, diverticulosis, is commonly observed in older individuals in Western societies but is less common outside of these countries. The triggers that lead from diverticulosis to diverticulitis remain unknown, and the incidence of diverticulitis appears to be increasing, both in the United States ${ }^{3}$ and in Europe. ${ }^{4}$ Recent studies have uncovered novel associations between diverticulitis and risk factors such physical activity ${ }^{5}$ and nonsteroidal anti-inflammatory drug use,${ }^{6}$ described possible genetic risk factors, ${ }^{7}$ and called into question older associations such as dietary fiber and nut and seed consumption. 8,9

Recently, a seasonal pattern of admissions for diverticulitis was described in the United States, with a consistent peak in admissions during the summer months. ${ }^{10}$ Seasonality is more pronounced in northern latitudes, which experience greater annual fluctuation in ultraviolet radiation. ${ }^{11}$ The etiology of this seasonal variation is unclear; dietary changes, dehydration, altered colonic motility, infectious processes, or seasonal flux in vitamin D may play a role. ${ }^{12}$ In this study, we examined whether the seasonality of diverticulitis is observed internationally and, in particular, whether this trend persists in the opposite seasons of the southern hemisphere.

\section{PATIENTS AND METHODS}

\section{Patient Population}

For this study, we used the Global Comparators (GC) database from Dr Foster Intelligence.

13 The GC database is a global hospital benchmarking collaborative (http://

drfosterintelligence.co.uk/global-comparators/). The GC data set contains all of the inpatient admissions from more than 50 participating hospitals in 10 countries, including diagnostic codes, demographic factors, urgency of admission, and procedures performed. Centrally, the submitted data are pooled, disparate coding systems combined, and quality controls applied. The goal of the GC database is to provide a central resource for participating institutions to reconcile differing coding systems into risk-adjustment models, which allows for useful and reliable international comparisons. Participating hospitals are generally large tertiary care centers. Most are teaching institutions. Government affiliation varies by country.

We included all of the inpatient admissions between 2008 and 2013 to participating hospitals in Australia (6 hospitals), the United Kingdom (10 hospitals), and the United States (10 hospitals). These countries were selected as those that had multiple hospitals contributing data to the GC data set throughout the entire study period. We excluded pediatric patients 
( $<18$ years of age), admissions for procedures of $<24$ hours, and low-quality records. The individual participating hospitals were the same throughout the study period and submitted data for the entire analyzed time frame. The hospitals were chosen for complete participation throughout the study period; not all of the current GC participants had data available for the entire time. Admissions, not discharges, were used as the primary data point. Nonelective admissions for acute diverticulitis were identified by the primary International Classification of Diseases-9 and International Classification of Diseases-10 codes for the admission (see Tables S1 and S2, Supplemental Digital Content, http:// links.lww.com/DCR/A240) in accordance with previously published work. ${ }^{11,12,14-16}$ Rates of urgent admission for diverticulitis were calculated against all of the adult admissions (both urgent and nonurgent) for participating hospitals in a particular country.

\section{Temporal Trends}

We tested for temporal trends in diverticulitis incidence using multiple methods. ${ }^{17,18}$ For visual interpretation, we first plotted a monthly mean for diverticulitis admissions. The monthly mean is a rate (nonelective admissions for diverticulitis per all admissions to hospital) that is normalized to the length of the month, such that all of the admissions are given equal weight and not miscounted by the varying lengths of months. We then created a cosinor model that assumes a parametric (sinusoidal) seasonal pattern of incidence. ${ }^{19}$ This model is necessarily symmetric around its peak; using this structure, it estimates the amplitude (height) and phase (time) of the seasonal variation.

To further understand changes over time, we used exponential time series to separate the seasonal trends from the year-to-year variation. We used the forecast package to select the best exponential time series model by Akaike information criterion. ${ }^{20}$ Exponential time series models are used to estimate the seasonal pattern by separating the overall trend (examining increases or decreases over time), its seasonal (cyclic) component, and the random error. This is accomplished by iteratively choosing different model fits to determine the best description of the overall trend and seasonal components; the randomness of the error was confirmed visually. Finally, we created separate logistic regression models for each country for the outcome of nonelective admission for diverticulitis and adjusted for age, sex, and comorbidity score with a focus on the individual months to measure seasonal trends. Initially, the month of February was chosen as a reference month to represent winter in the United Kingdom and the United States; to further clarify the opposite seasonal pattern, a separate set of models was created by only changing the reference month to August, such that the reference was now shifted to Australian winter.

\section{Statistical Analysis and Institutional Review Board Approval}

Data were analyzed using R 3.1.2 (R Core team, Vienna, austria). all of the results are reported with $95 \%$ Cis or $p$ values, as appropriate. the threshold for significance was 2-tailed $p<0.05$. institutional review board approval was granted by massachusetts General hospital. 


\section{RESULTS}

\section{Patient and Hospital Characteristics}

In our database, a total of 5318 patients were urgently admitted with diverticulitis in Australia, 8737 in the United Kingdom, and 4617 in the United States (Table 1). The majority were women (52.2\% in Australia, 60.8\% in the United Kingdom, and 57.3\% in the United States), with a mean age in the seventh decade (60.7 years in Australia, 67.0 years in the United Kingdom, and 60.1 years in the United States). Clinically, a comparable number of patients underwent a surgical procedure, with $11.9 \%$ in Australia, $15.8 \%$ in the United Kingdom, and $16.0 \%$ in the United States. The mean length of stay for all of the patients was 6.3 days in Australia, 8.4 days in the United Kingdom, and 5.7 days in the United States. All of these differences were statistically significant $(p<0.001$ for all).

A total of 5.5-million hospital admissions were included in our database. For the 6 hospitals in Australia, there were more than 1.2-million admissions (Table 2). The 10 United Kingdom hospitals contributed 2.7-million admissions, and the 10 hospitals in the United States consisted of 1.6-million admissions. Average hospital volume was highest in the United Kingdom $(45,233$ admissions annually), followed by Australia $(35,132)$ and the United States $(29,492)$.

\section{Seasonal Patterns in Diverticulitis Admissions}

Four different analytic methods were used to assess seasonality in these data. First, we plotted the raw incidence (normalized to 10,000 hospital admissions) of admissions for acute diverticulitis in all 3 of the countries (Figs. 1A, C, and E). Once normalized to the monthly mean, a clear visual pattern emerged (Figs. 1B, D, and F). There was a clear peak in Australia in the early part of the year, with April being the highest month. In the United Kingdom, the difference was smaller, but the peak was in September. In the United States, that peak was in August.

Because a clear trend was observed, we chose to use classic logistic regression with a special focus on the month of admission to test for trends (Table 3). Adjusting for age, sex, and year of admission, and using February as the reference month, the Australian winter months of June, July, and August were associated with a lower odds of hospital admission for diverticulitis. In the United Kingdom, the months of August, September, and October were associated with significantly higher odds of admission for diverticulitis, and this was true in the US summer months of May, June, July, August, and September. To aid in understanding the opposite pattern, a separate set of logistic regressions was created with August as the reference month, which represents Australian winter (see Table S3, Supplemental Digital Content, http://links.lww.com/DCR/A240). With August as the reference month, there was a significantly higher odds of hospital admission for diverticulitis in December through May for Australia; the mirror pattern of lower odds of admission was found in the United Kingdom for November through June and in the United States for December through February.

We then moved to analyze with other formal hypothesis testing of periodicity. When the data were modeled parametrically, a statistically significant trend was observed (Table 4). In 
these cosinor models, a sinusoidal wave is fit to the data that cycle over the year, creating a parametric description. From that model, the amplitude and peak month can be ascertained, and a null hypothesis of no association is used. The amplitude describes the height of the peak, and the peak month (from the models) correlates with the numerical month in the cycle (range, 1-12). Under this model, Australia had the largest amplitude (6.2) with a peak in February during Australian summer (2.0), whereas the peak months for the United Kingdom (7.8) and the United States (7.0) corresponded with July, during summer in the Northern Hemisphere. As a marker of illness severity, there was no difference in median length of stay by month for Australia ( $p=0.24$ ) or the United Kingdom $(p=0.65)$. However, the median length of stay was different in the United States, where it was 3 days in September and October; all of the other months were $4(p=0.03)$.

Exponential time series decomposition models revealed similar findings (Fig. 2). All were chosen to be an additive error model by Akaike information criterion. A nonparametric description of the seasonal variation is first described, and then this pattern is subtracted from the observed data to extract any trend over time. For all 3 of the countries (Australia Fig. 2A, the United Kingdom Fig. 2B, and the United States Fig. 2C), there was a slight increase in admissions over time. The seasonal descriptions still have clear peaks and valleys, and the errors are randomly distributed around the baseline, suggesting, again, that there is a seasonal pattern to these data.

\section{Sensitivity Analyses}

Because there were some patients readmitted for diverticulitis in the study period, we performed sensitivity analyses excluding those patients: 666 (12.5\%) in Australia, 1045 (11.9\%) in the United Kingdom, and 454 (9.8\%) in the United States. When these patients were excluded, the findings were largely the same. There were no significant changes to the baseline demographics. The cosinor models all remained significant at $p<0.025$. Similarly, the logistic regression to measure month-by-month changes had the same months, with the addition of September, October, and November for Australia.

\section{DISCUSSION}

In this study, we demonstrate that the summer peak in diverticulitis admission is observed consistently in geographically distinct populations across the equator. By using various models, we found that a consistent seasonal pattern does exist with regard to hospital admission for acute diverticulitis. These data suggest the existence of a shared seasonal risk factor for diverticulitis between these populations. Seasonal changes in diet, physical activity, or medication use may trigger diverticulitis. Alternatively, a seasonal pathogen may trigger the onset of the condition. Alternatively, seasonal swings in UV radiation may influence diverticulitis, possibly mediated via vitamin D. An additional alternative is that relative dehydration in the summer slows colonic transit, resulting in fecal stasis and potentially increasing the susceptibility to diverticulitis. In light of our current study, we favor a non-behavioral risk factor, because it seems unlikely that seasonal behavioral changes would be shared robustly over global populations. Other risk factors for diverticular 
disease, such as age, race, and obesity, may contribute to the overall frequency of diverticular disease but do not vary by season.

We identified statistically significant differences in the age, sex, and comorbidity severities among international populations, although in all of the countries patients with diverticulitis were $>60$ years of age and the majority were women. In addition, length of stay, readmission rates, and rates of surgery varied among countries. Compared with Australia and the United Kingdom, the United States demonstrated higher rates of surgery, shorter lengths of stay, and decreased readmissions. We are cautious in drawing clinical conclusions from these data because there is likely a strong influence of practice differences and healthcare system variables. Again, these practice and healthcare system factors are unlikely to vary by season. Using length of stay as a surrogate marker for disease severity, we did not find a longer length of stay in the summer months for any population.

The strengths of our study include its international approach, multiple statistical modeling methodologies, and the robust quality control applied to the data, allowing for meaningful comparisons among populations. In addition, the magnitude of the seasonal change (22\%$24 \%$ maximal increase in admissions during peak months) is similar to the $16 \%$ to $25 \%$ summer increase in diverticulitis admissions observed in analyses of the US Nationwide Inpatient Sample. ${ }^{10,11}$ However, this study is subject to the limitations of deidentified database research. These are magnified by international differences in billing coding (International Classification of Diseases-10 in the United Kingdom and Australia versus International Classification of Diseases-9 in the United States), although central data quality control is a feature of the GC data set. Diagnoses are determined by billing coding and subject to inaccuracy, although these errors are unlikely to vary by season. In addition, analysis is limited to the participating hospitals in the GC data set. These hospitals represent a small fraction of the hospitals within their respective countries but compose the only combined, quality-controlled international data set of inpatient admissions to date. The observation of seasonality within this small data set may speak to the magnitude of this variation. However, the small data set precludes calculation of a true incidence and may not generalize to national trends. Nonetheless, given the detection of this seasonal trend within this relatively small data set, across multiple databases and multiple countries, we believe that it represents a real epidemiologic phenomenon. An additional limitation is the restriction of episodes of diverticulitis to inpatient admissions, which neglects uncomplicated episodes managed in the outpatient setting. However, outpatient episodes are difficult to reliably identify and may be less likely to represent true bouts of diverticular disease. Also, although this study involves multiple countries in both the Northern and Southern Hemispheres, the Dr Foster Intelligence database that we relied on currently includes only developed countries, and the generalizability of our findings to other countries is unknown, although this could be investigated in the future as the Dr Foster Intelligence database grows and additional countries are added to its database.

In addition to the findings regarding diverticulitis, this study also provides a model for robust identification and analysis of trends from noisy data and demonstrates that billing databases, when properly analyzed, can not only investigate healthcare delivery and quality but generate insights into the mechanisms of disease. Furthermore, although the current study 
involves epidemiological data at the population level, the analytical techniques could be applied to other big data at the patient level, as well to identify trends from other noisy timetrend data. For example, given technological advances in patient monitoring, the methodologies demonstrated in this study can be useful to detect patterns of heart rate variability from intensive care unit monitors, to monitor patterns of weight loss over time after bariatric surgery, or to monitor fluctuations in patient quality of life after surgery.

\section{CONCLUSION}

This study demonstrates that a seasonal summer increase in diverticulitis admissions is present internationally and in both hemispheres. Although common, diverticulitis is incompletely understood, and recent research has called into question traditional theories regarding its etiology, natural history, and optimal treatment. This study suggests that a shared seasonal risk factor is present across international populations and may provide a stimulus for additional needed research into the pathophysiology of this common disease.

\section{Supplementary Material}

Refer to Web version on PubMed Central for supplementary material.

\section{ACKNOWLEDGMENTS}

The authors thank Rose Higgins of Dr Foster Intelligence for her invaluable assistance with additional data analysis.

Funding/Support: Dr Adler is funded by the Arthur Tracy Cabot Fellowship from the Center for Surgery and Public Health at Brigham and Women's Hospital.

\section{REFERENCES}

1. Peery AF, Dellon ES, Lund J, et al. Burden of gastrointestinal disease in the United States: 2012 update. Gastroenterology 2012;143:1179-1187.e1. [PubMed: 22885331]

2. Painter NS, Burkitt DP. Diverticular disease of the colon: a deficiency disease of Western civilization. Br Med J 1971;2:450-454. [PubMed: 4930390]

3. Wheat CL, Strate LL. Trends in hospitalization for diverticulitis and diverticular bleeding in the United States from 2000 to 2010. Clin Gastroenterol Hepatol 2016;14:96-103.e1. [PubMed: 25862988]

4. Jamal Talabani A, Lydersen S, Endreseth BH, Edna TH. Major increase in admission- and incidence rates of acute colonic diverticulitis. Int J Colorectal Dis 2014;29:937-945. [PubMed: 24802814]

5. Strate LL, Liu YL, Aldoori WH, Giovannucci EL. Physical activity decreases diverticular complications. Am J Gastroenterol 2009;104:1221-1230. [PubMed: 19367267]

6. Strate LL, Liu YL, Huang ES, Giovannucci EL, Chan AT. Use of aspirin or nonsteroidal antiinflammatory drugs increases risk for diverticulitis and diverticular bleeding. Gastroenterology 2011;140:1427-1433. [PubMed: 21320500]

7. Strate LL, Erichsen R, Baron JA, et al. Heritability and familial aggregation of diverticular disease: a population-based study of twins and siblings. Gastroenterology 2013;144:736-742.e1; quiz e14. [PubMed: 23313967]

8. Strate LL, Liu YL, Syngal S, Aldoori WH, Giovannucci EL. Nut, corn, and popcorn consumption and the incidence of diverticular disease. JAMA 2008;300:907-914. [PubMed: 18728264]

9. Peery AF, Barrett PR, Park D, et al. A high-fiber diet does not protect against asymptomatic diverticulosis. Gastroenterology 2012;142:266-272.e1. [PubMed: 22062360] 
10. Ricciardi R, Roberts PL, Read TE, et al. Cyclical increase in diverticulitis during the summer months. Arch Surg 2011;146:319-323. [PubMed: 21422363]

11. Maguire LH, Song M, Strate LL, Giovannucci EL, Chan AT. Association of geographic and seasonal variation with diverticulitis admissions. JAMA Surg 2015;150:74-77. [PubMed: 25409019]

12. Maguire LH, Song M, Strate LE, Giovannucci EL, Chan AT. Higher serum levels of vitamin D are associated with a reduced risk of diverticulitis. Clin Gastroenterol Hepatol 2013;11:1631-1635. [PubMed: 23954650]

13. Bottle A, Middleton S, Kalkman CJ, Livingston EH, Aylin P. Global comparators project: international comparison of hospital outcomes using administrative data. Health Serv Res 2013;48(6 pt 1):2081-2100. [PubMed: 23742025]

14. Morris CR, Harvey IM, Stebbings WS, Hart AR. Incidence of perforated diverticulitis and risk factors for death in a UK population. Br J Surg 2008;95:876-881. [PubMed: 18509877]

15. Kawatkar A, Chu LH, Iyer R, et al. Development and validation of algorithms to identify acute diverticulitis. Pharmacoepidemiol Drug Saf 2015;24:27-37. [PubMed: 25257514]

16. Shahedi K, Fuller G, Bolus R, et al. Long-term risk of acute diverticulitis among patients with incidental diverticulosis found during colonoscopy. Clin Gastroenterol Hepatol 2013;11:16091613. [PubMed: 23856358]

17. Barnett AG, Baker P, Dobson A. Analysing seasonal data. R Journal 2012;4:5-10.

18. Rau R. Seasonality in Human Mortality New York, NY: Springer Science \& Business Media; 2006.

19. Barnett AG, Dobson AJ. Analysing Seasonal Health Data New York, NY: Springer Science \& Business Media; 2010.

20. Hyndman R, Koehler AB, Ord JK, Snyder RD. Forecasting With Exponential Smoothing New York, NY: Springer Science \& Business Media; 2008. 


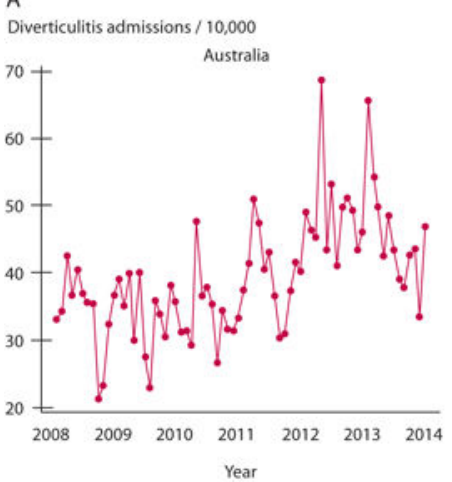

C

Diverticulitis admissions $/ 10,000$

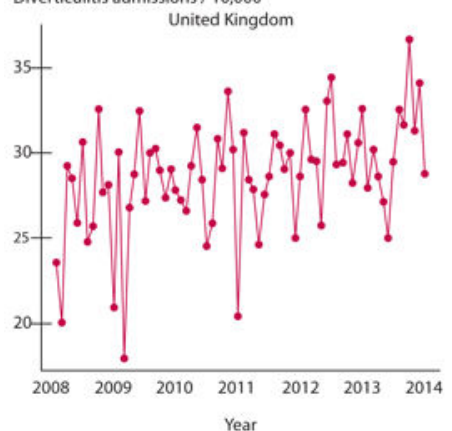

E

Diverticulitis admissions / 10,000

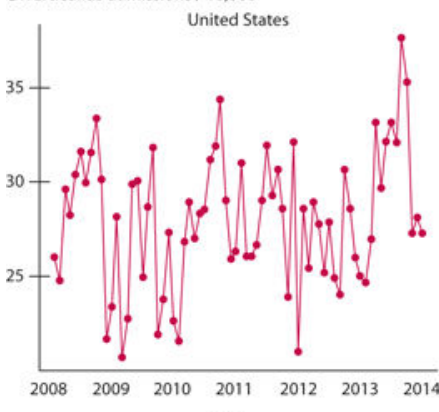

Year
B

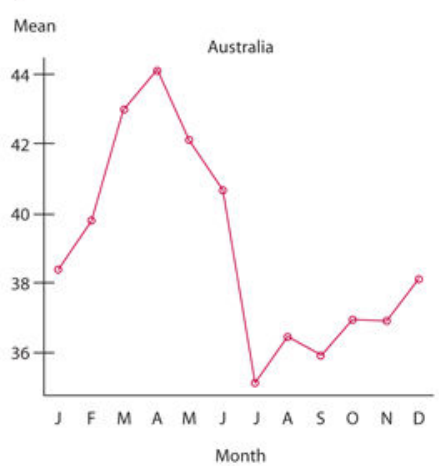

D

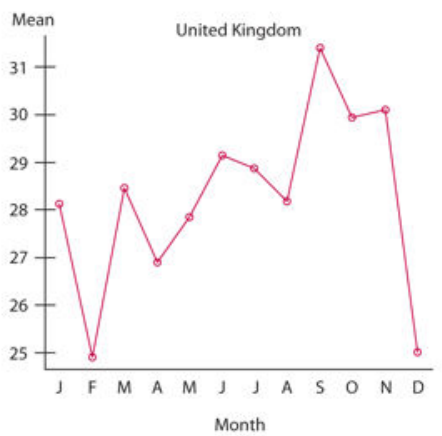

F

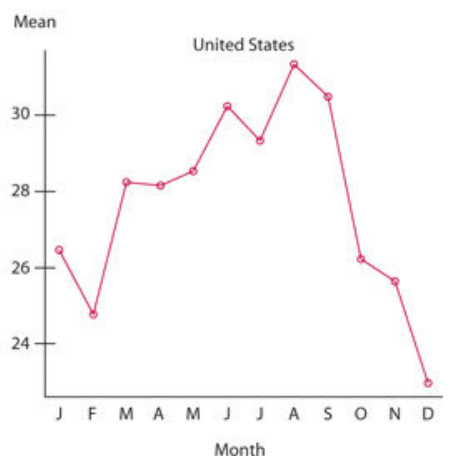

FIGURE 1.

A, C, and E represent the prevalence data for each country. Each dot represents 1 month over the course of the year. B, D, and F are the monthly mean for each country, which is a normalized plot that combines all similar months and accounts for the different lengths of months.

Dis Colon Rectum. Author manuscript; available in PMC 2018 September 13. 

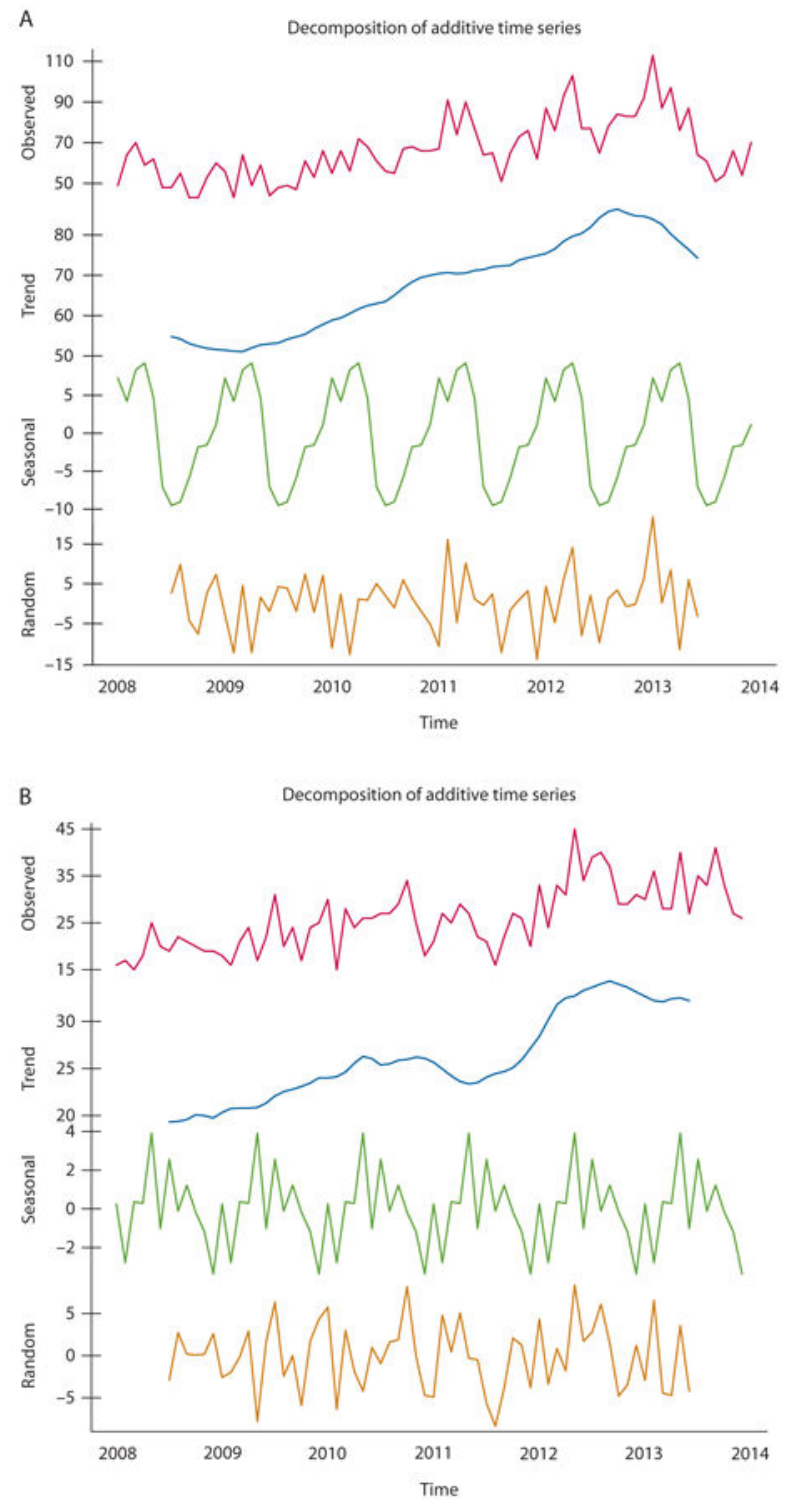

FIGURE 2.

Exponential time series decomposition models. After selection by Akaike information criteria, all 3 countries (A: Australia, B: United Kingdom, and C: United States) were best described by an additive time series. Once the seasonal trends (seasonal) were modeled and extracted, all showed a slight increase in the number of admissions for diverticulitis (trend). 
TABLE 1.

Patients admitted with diverticulitis, characteristics by country

\begin{tabular}{lcccc}
\hline Variable & Australia & United Kingdom & United States & $p$ \\
\hline Total patients, n & 5318 & 8737 & 4617 & $<0.001$ \\
Age, y & $60.7 \pm 16.3$ & $67.0 \pm 16.1$ & $60.1 \pm 15.9$ & $<0.001$ \\
Women & $2775(52.2)$ & $5308(60.8)$ & $2647(57.3)$ & $<0.001$ \\
Comorbidity score & $6.0 \pm 13.9$ & $7.6 \pm 14.0$ & $10.2 \pm 19.2$ & $<0.001$ \\
Surgery & $633(11.9)$ & $1377(15.8)$ & $737(16.0)$ & $<0.001$ \\
Length of stay & $6.3 \pm 11.2$ & $8.4 \pm 12.4$ & $5.7 \pm 6.6$ & $<0.001$ \\
Readmission within 30 d & $343(6.4)$ & $920(4.9)$ & $502(2.7)$ & $<0.001$
\end{tabular}

Values are presented as mean \pm SD or $\mathrm{n}(\%)$. 
TABLE 2.

Hospital characteristics by country

\begin{tabular}{lccc}
\hline Variable & Australia & United Kingdom & United States \\
\hline Number of hospitals & 6 & 10 & 10 \\
Total admission volume & $1,264,744$ & $2,713,976$ & $1,622,979$ \\
Average annual volume & $210,791 \pm 9993$ & $452,329 \pm 4442$ & $270,347 \pm 12,939$ \\
Average annual hospital volume & $35,132 \pm 10,692$ & $45,233 \pm 17,755$ & $29,492 \pm 13,641$ \\
\hline
\end{tabular}

Values are characterized as mean $\pm \mathrm{SD}$. 
TABLE 3.

Logistic regression for month trends in diverticulitis admission incidence

\begin{tabular}{|c|c|c|c|c|c|c|}
\hline \multirow[b]{2}{*}{ Month } & \multicolumn{2}{|l|}{ Australia } & \multicolumn{2}{|c|}{ United Kingdom } & \multicolumn{2}{|c|}{ United States } \\
\hline & OR $(95 \% C I)^{a}$ & $p$ & OR $(95 \% C I)$ & $p$ & OR $(95 \% C I)$ & $p$ \\
\hline January & $1.03(0.91-1.18)$ & 0.62 & $1.03(0.92-1.14)$ & 0.61 & $1.06(0.92-1.23)$ & 0.42 \\
\hline February & Reference & & Reference & & Reference & \\
\hline March & $0.98(0.87-1.11)$ & 0.78 & $1.06(0.96-1.18)$ & 0.25 & $1.13(0.98-1.31)$ & 0.10 \\
\hline April & $1.05(0.93-1.19)$ & 0.45 & $1.06(0.95-1.18)$ & 0.28 & $1.13(0.97-1.31)$ & 0.12 \\
\hline May & $0.94(0.83-1.07)$ & 0.34 & $1.08(0.97-1.20)$ & 0.16 & $1.17(1.01-1.36)$ & 0.03 \\
\hline June & $0.83(0.72-0.94)$ & $<0.001$ & $1.07(0.96-1.19)$ & 0.21 & $1.18(1.02-1.37)$ & 0.02 \\
\hline July & $0.78(0.68-0.89)$ & $<0.001$ & $1.10(0.99-1.23)$ & 0.06 & $1.17(1.02-1.36)$ & 0.03 \\
\hline August & $0.77(0.68-0.89)$ & $<0.001$ & $1.22(1.10-1.35)$ & $<0.001$ & $1.24(1.08-1.44)$ & $<0.001$ \\
\hline September & $0.88(0.77-1.00)$ & 0.05 & $1.16(1.04-1.28)$ & 0.01 & $1.24(1.07-1.43)$ & $<0.001$ \\
\hline October & $0.90(0.79-1.02)$ & 0.10 & $1.16(1.04-1.28)$ & 0.01 & $1.11(0.96-1.29)$ & 0.15 \\
\hline November & $0.88(0.77-1.01)$ & 0.07 & $1.04(0.94-1.16)$ & 0.42 & $1.11(0.96-1.29)$ & 0.17 \\
\hline December & $1.00(0.88-1.14)$ & 1.00 & $0.94(0.84-1.05)$ & 0.27 & $1.01(0.86-1.17)$ & 0.92 \\
\hline
\end{tabular}

${ }^{a}$ ORs represent the ratio of probabilities that any given month will contain an admission in comparison with February, which represents summer in Australia and with winter in United Kingdom and the United States. ORs were adjusted for age, sex, and year of admission. 
TABLE 4.

\section{Periodicity/trend test}

\begin{tabular}{lccc}
\hline Country & Amplitude & Peak month & $\begin{array}{l}\text { Significant for } \\
\text { sinusoidal pattern }\end{array}$ \\
\hline Australia & 6.2 & 2.0 & Yes \\
United Kingdom & 1.9 & 7.8 & Yes \\
United States & 2.1 & 7.0 & Yes \\
\hline
\end{tabular}

Cosinor models were fit to the data, modeling a sinusoidal pattern with a peak month (range, 1-12) along with a relative amplitude. Significance is at $p<0.025$. 